\title{
Applications of nanowire arrays in nanomedicine
}

Berthing, Trine; Sørensen, Claus Birger; Nygård, Jesper; Martinez, Karen Laurence

Published in:

Journal of Nanoneuroscience

DOI:

10.1166/jns.2008.001a

Publication date:

2009

Citation for published version (APA):

Berthing, T., Sørensen, C. B., Nygård, J., \& Martinez, K. L. (2009). Applications of nanowire arrays in nanomedicine. Journal of Nanoneuroscience, 1, 3-9. https://doi.org/10.1166/jns.2008.001a 


\title{
Applications of Nanowire Arrays in Nanomedicine
}

\author{
Trine Berthing ${ }^{1}$, Claus B. Sørensen², Jesper Nygård², and Karen L. Martinez ${ }^{1, *}$ \\ ${ }^{1}$ Bio-Nanotechnology Laboratory, Department of Neuroscience and Pharmacology \& Nano-Science Center, \\ University of Copenhagen, Universitetsparken 5, DK-2100, Copenhagen, Denmark \\ ${ }^{2}$ Niels Bohr Institute \& Nano-Science Center, University of Copenhagen, Universitetsparken 5, DK-2100, Copenhagen, Denmark
}

\begin{abstract}
In recent years, the potential of nanowires for applications within nanomedicine, including drug delivery and diagnostics, have been explored by several groups. The small diameter of nanowires allows them to cross the lipid bilayer of the cell membrane without causing significant damage, which makes them attractive as a tool for gaining access to the intracellular environment. Recent studies report on an approach using vertically aligned nanowires fixed to a surface instead of nanowires in solution. Nanowire arrays hold promise of simpler maneuvering and additional applications within drug delivery, biosensing, and electrophysiology in living cells, all of which are explored in this paper.
\end{abstract}

Keywords: Nanowires, Drug Delivery, Biosensor, Electrophysiology, Nanoelectrode, Nanorod, Nanofiber, Nanomedicine.

\section{INTRODUCTION}

The emerging field of nanomedicine has been propelled by the growth and gradual overlap of disciplines such as nanotechnology, biochemistry, pharmacology, and medicine, all of which are themselves highly interdisciplinary. The new field of nanomedicine integrates advanced nanomaterials such as nanowire (NW) arrays with biomolecules, cells, and eventually organisms, in order to develop novel platforms for applications such as biosensing, drug screening, and drug delivery.

In biochemistry, developments in genomics and proteomics have helped elucidate the molecular basis of many diseases. They have generated knowledge about disease biomarkers, with potential for personalized medicine in diagnostics, in which fine therapies (drug type, dose) can be adapted to each patient, reducing side effects and improving drug efficiency.

Drug discovery has also benefited from these developments with the design of new drugs and the development of highthroughput screening (HTS) strategies for new molecules. Combined with drug delivery strategies based on novel nanomaterials, with better biocompatibility and target specificity, nanomedicine opens the way to a myriad of potential new therapies (Cheng et al., 2006).

Based on these developments, there are three main impact areas foreseen for nanomedicine:

(1) high-quality and fast diagnosis of diseases, with the development of biosensors;

(2) drug discovery, with the development of fast and cheap screening strategies of potential new drugs; and

(3) drug delivery, with targeted delivery and translocation of active therapeutic reagents.

Neuroscience raises additional challenges that can also benefit from the development of novel nanotechnology-based platforms.

*Author to whom correspondence should be addressed.
These challenges stem in particular from the regulation of the brain microenvironment through the blood-brain barrier and the organization of neurons and their specific response to a panel of neurotransmitters.

The use of carbon nanotubes (CNTs) for the delivery of therapeutic agents has recently been demonstrated, with applications for the delivery of genes or vaccines, and with potential for higher-efficiency release of molecules used in cancer and AIDS therapies (Lacerda et al., 2007). Recently, a couple of laboratories (Hällström et al., 2007; Kim et al., 2007) reported on an alternative method of interfacing NWs and cells that holds promise of simpler maneuvering and additional applications. Instead of working with solutions of NWs, suspensions of living cells are added to an array of vertically aligned NWs that are fixed to a surface. Like CNTs, the NW arrays present a major potential in nanomedicine, with possible applications for drug delivery and discovery. In addition, these arrays could provide a huge potential for diagnostics in living cells.

\section{Nanowires-Structures and Properties of Relevance for Biological Applications}

NWs are solid, crystalline rods with diameters in the nanometer range $(10-100 \mathrm{~nm})$ and typical lengths of around a few micrometers. These objects can be made from a wide range of inorganic materials ( $\mathrm{Lu}$ et al., 2006; Wu et al., 2002) and are occasionally termed nanorods or nanowhiskers. In contrast, CNTs are hollow, cylindrical molecules made purely from carbon.

The growth of linear crystals is promoted by the presence of nanoscale metal catalyst particles on a surface. At elevated temperatures the catalyst particles act as ultrafine melting pots, consisting of the molten metal particle and semiconductor atoms supplied from a dilute gas in the growth chamber. When this alloy is saturated, a fine crystalline wire grows out from the particle 

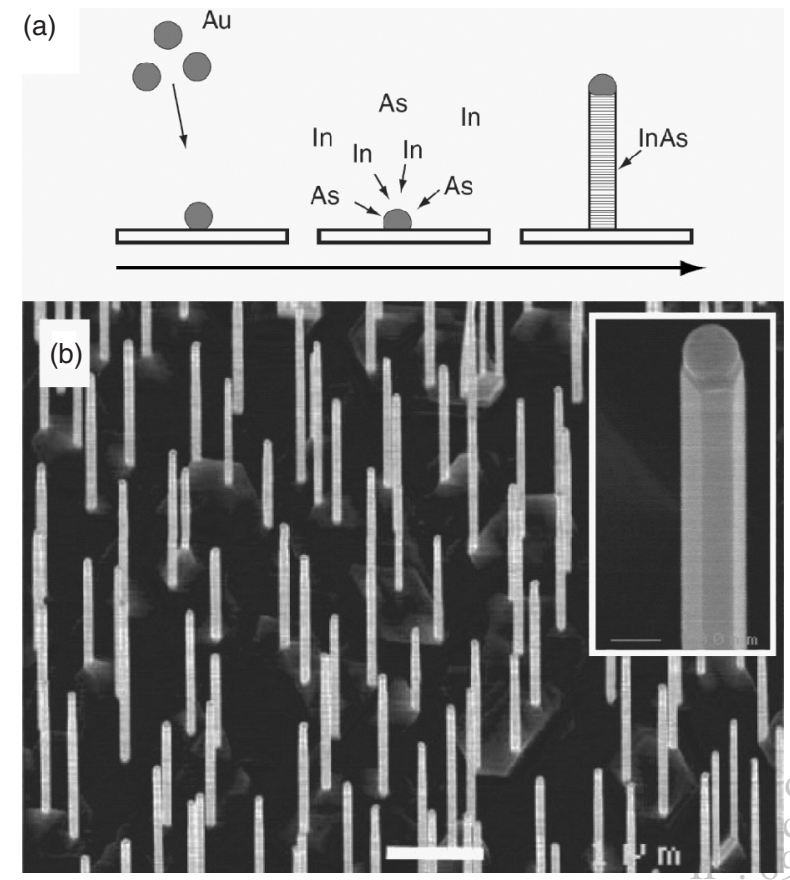

FIG. 1. (a) Growth model for NWs. Gold (Au) catalyst nanoparticles are deposited on a surface. At elevated temperatures, atoms from the surrounding gas (in this example, indium [In] and arsenic [As]) assemble into a linear crystal underneath each catalyst particle. (b) Scanning electron micrograph of an aligned NW array of indium-arsenide (InAs), scale bar $1 \mu \mathrm{m}$. Inset shows a zoom on a single NW with the gold particle remaining at the top, scale bar $100 \mathrm{~nm}$. (Courtesy of M. Aagesen, University of Copenhagen.)

and lifts it from the surface (Fig. 1(a)). This growth procedure results in a surface covered with a forest of vertically aligned NWs. The diameter of a wire is determined by the size of catalyst particle; wires with narrow diameter distributions can be grown through proper preparation of the catalyst.

The density and positions of NWs on the surface is determined by the initial distribution of the catalyst particles. In Figure 1(b) catalyst particles were deposited randomly on the surface prior to growth, resulting in a disordered pattern. However, it is possible to predefine exactly the positions of the NWs by arranging the catalyst particles in any desired pattern prior to growth using lithographic methods. (Mårtensson, 2004). Ordered arrays can also be made from CNTs and nanofibers and are commercially available.

Usually NWs are harvested from the surface after growth and used in solution or deposited onto a new surface. However, the focus of the present paper is to explore the applications of NWs that have been left standing on the surface (from here on referred to as $N W$ arrays). An example of such a NW array is shown in Figure 1(b).

Under well-controlled conditions, large areas of wires with uniform properties, in both their physical dimensions and their intrinsic properties (crystal structure, electrical and optical properties) (Li et al., 2006; Lu et al., 2006; Thelander et al., 2006) can be grown. The latter is given solely by their elemental composition; i.e., wires made from semiconductor materials like silicon and germanium (group IV elements) or galliumarsenide and indium-phosphide (group III-V) will indeed be semiconducting. CNTs can possess different electrical properties, depending on their microscopic geometry, and thus an ensemble of nanotubes will contain a random distribution of metallic and semiconducting tubes. For applications based on their electrical properties this is a nuisance, but structurally it makes no significant difference.

In addition to their natural properties and geometry that make them attractive for biological applications, semiconductor NWs can be finely tailored, which opens a large number of options in their application to biological material: They can be doped with minute concentrations of other elements, whereby their conductivity can be adjusted, in contrast to metallic wires. In addition, their elemental composition can be changed along the wire during growth, forming so-called heterostructures (Lauhon et al., 2004). This opens possibilities for functionalizing various parts of the NWs selectively according to a design defined during growth. Finally, the surface of NWs can be customized with coatings of protective or active layers, either by making core-shell structures directly during growth or by post-treatments. Most importantly, their surfaces can be chemically modified, allowing a wide range of functionalizations (Patolsky et al., 2006).

The interest in semiconductor NWs was initially not for biological/applications, but mainly due to their favorable electronic and optical properties. For instance, high-performance nanoscale transistors (field-effect transistors, or FETs) can be made from semiconductor NWs (NW-FETs), making it plausible that nanoscale electronic circuits can be based on these wires ( $\mathrm{Li}$ et al., 2006; Thelander et al., 2006). In the context of nanomedicine is it interesting to note that ultrasensitive biosensors can be made from NW-FETs by properly functionalizing their surface (Patolsky et al., 2006). Such sensors have direct electrical readout and offer label-free detection, as also demonstrated for CNT-based biosensors (Kumar, 2007). Recent publications also demonstrate the potential of arrays of NWs for applications in living cells, which is the scope of this paper.

\section{Why are NW Arrays Attractive for Applications with Living Cells?}

The most apparent motivation for looking at applications of NWs in nanomedicine is that their dimensions are compatible with the dimensions of mammalian cells, with membrane thickness of 4-5 $\mathrm{nm}$ and diameters between 1 and $20 \mu \mathrm{m}$. The small diameter of the NWs allows them to cross the lipid bilayer of the cell membrane without causing significant damage (Lacerda et al., 2007). By comparison, micropipettes commonly used as microinjectors or for electrophysiology measurements on cells have a diameter of about $1 \mu \mathrm{m}$, which is up to 100 times larger, so based on geometry NWs are potentially less invasive than existing tools.

At the same time, the height of the wires can be adjusted for the purpose of adapting the array to cell types of different dimensions or for bringing different parts of cells within reach. NWs of less than $1 \mu \mathrm{m}$ would cross the cell membrane and reach into the cytosol close to the membrane, whereas longer wires of several micrometers could possibly penetrate into the cell nucleus or other compartments. Wires could even be long enough to enter a cell on one side and exit on the other.

The expected limited invasiveness of the NWs due to their dimensions is not their only advantage. There are also a number of 
important advantages stemming from the fact that they are grown on surfaces with controlled distribution and orientation. First of all, it is possible to control the position of the NWs via photolithography. This allows organization of the NWs into arrays of various designs. Moreover, NW density can be controlled, and this allows for tuning the roughness of the surface, and most importantly, the number of NWs to be inserted into each cell.

In the last few years there has been considerable progress in the design of vertically aligned NWs for the purpose of interfacing them with cells, primarily represented by the work done by McKnight et al. (Guillorn et al., 2002; McKnight, Melechko et al., 2006; McKnight, Peeraphatdit et al., 2006). As an example, the NW array can be marked with an indexed grid, which facilitates tracking of specific positions in the array, making it possible to record time series of specific cells (McKnight et al., 2004).

For the reasons mentioned earlier, it should be possible to create a platform with an ideal design; i.e., a customized array of vertically aligned NWs, with defined orientation, length, and diameter, providing a support with controlled surface roughness, which is a crucial parameter for cell adhesion, and with a predefined number of probes per cell.

\section{Arrays of Nanowires Incorporated in Living Cells}

The first evidence that it is possible to interface living cells with an array of vertically aligned nanostructures was given in 2003 by McKnight et al. Here an array of conically shaped carbon nanostructures with tip diameters in the range of $30 \mathrm{~nm}$ and lengths of $7 \mu \mathrm{m}$ were functionalized with plasmid DNA encoding green fluorescent protein (GFP). Chinese hamster ovary (CHO) cells were forced onto this array by centrifugation and by subsequently applying pressure. Scanning electron micrographs revealed that the $\mathrm{CHO}$ cells were penetrated by the nanostructures, but only a transfection efficiency of less than $1 \%$ was achieved. Despite the penetrating structures and the violent treatment of the cells, the cells survived and proliferated for several days, indicating that the penetrating wires did not undermine the cell integrity.

A different approach was presented in the recent publications of Kim et al. (2007) and Hällström et al. (2007), in which NWs fixed to a surface penetrate cells without the use of an external force.

Kim et al. cultured human embryonic kidney (HEK293) cells and mouse embryonic stem (mES) cells directly on an array of silicon NWs. One hour after deposition of cell suspension the wires were shown to penetrate the cells. The viability was seen to increase with decreasing diameter of the NWs, consistent with the intuition that a smaller diameter will create less stress when penetrating the lipid bilayer.

Similar observations were made by Hällström et al. on mouse neurons cultured on a surface of vertically aligned galliumphosphide (GaP) NWs. The neurons had an even better adherence and a higher viability on a GaP-NW surface compared to a planar GaP surface. Although it is surprising that cells should have higher viability on a surface of nanoneedles, it could be an indication that the roughness of the nanostructured surface improves the adherence and thereby the viability, as seen for some other mammalian cell types (Khang et al., 2007; Kunzler et al., 2007).

In addition, we recently observed the incorporation of nonhomogeneous arrays (diameter 70-270 nm) of indium-arsenide (InAs) NWs in HEK293 cells as illustrated in Figure 2, without alteration of the cell integrity (Berthing, unpublished).
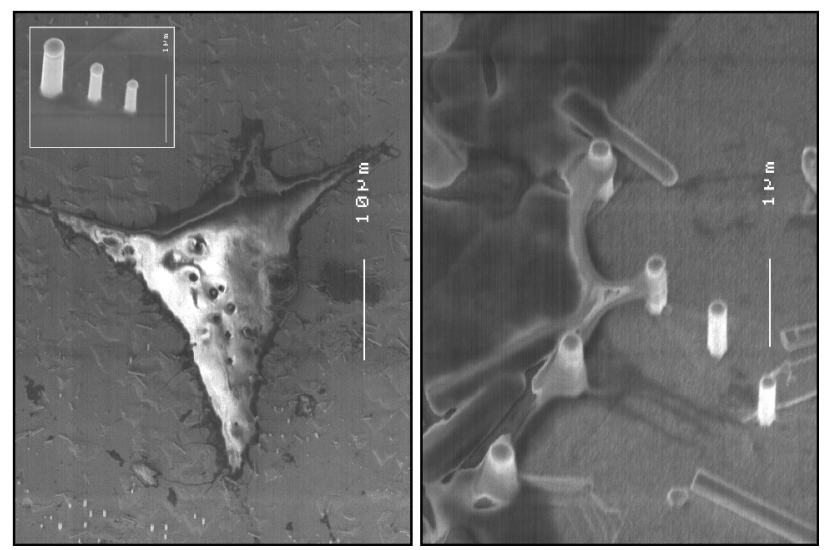

FIG. 2. (Left) Scanning electron micrograph of HEK293 cell cultured on an InAs NW array, scale bar $10 \mu \mathrm{m}$. Insert is a zoom on the NW array, scale bar $1 \mu \mathrm{m}$. (Right) InAs NWs embedded in HEK293 cell membrane, scale bar $1 \mu \mathrm{m}$.

This new approach seems attractive because of the simple protocol of using the NW array as a cell culture surface, but it might be a problem to apply it to more sensitive cell lines that have specific requirements to the culturing conditions. As an alternative, it would be interesting to be able to insert a NW array in a population of cells cultured on standard cell culture dishes or even in cells in tissue samples.

The mechanism of cell penetration by the wires is not yet known. Possible mechanisms could be a mechanical penetration occurring simply when the cells are settling onto the surface, or an active internalization through endocytosis. In the studies by Kim et al. and Hällström et al. it was observed that NWs in contact with cells were bent, which indicates that the cells exert a force on the NWs. This could possibly be due to an interaction between the wires and the cytoskeleton and might support the hypothesis of an active uptake of the wires by the cellular machinery. Taking advantage of cellular mechanics for the uptake of NWs is preferable for future applications of NW arrays because it most likely is less invasive, and it excludes the need of applying an external force.

The potential for widespread applications of NWs as intracellular probes is indicated by the various cell types that have been shown to be viable for several days when penetrated by NWs of different materials and geometry (Table 1). The cell types that have been tested span a range of important classes like epithelial cells, neurons, embryonic cells, and cancer cells, suggesting that the applications discussed in this text can be adapted to a variety of cell types and eventually tissues. Remarkably, even nonadherent cell lines can be interfaced with a NW array (McKnight et al., 2004).

The longest survival time of cells cultured on a NW surface is reported by McKnight et al. (2004), in which CHO cells survive more than three weeks on a silicon surface with vertically aligned carbon NWs (Table 1). These observations show the potential of arrays of NWs for long-term investigations of cells, which is a crucial parameter for the design of future applications.

There have been numerous investigations of the cytotoxicity of NWs and nanotubes in solution that have been taken up by cells (Cai et al., 2007). Oxidative stress (Manna et al., 2005), reduced respiratory activity (Jia et al., 2005), and enzyme inhibition (Yi et al., 2007) are some of the risks mentioned, but the cytotoxic 
TABLE 1. Overview of the wide range of cell types that have been interfaced with NW arrays.

\begin{tabular}{|c|c|c|c|c|c|}
\hline \multirow[b]{2}{*}{ Cell types } & \multicolumn{3}{|c|}{ NW properties } & \multirow[b]{2}{*}{ Min. viability (days) } & \multirow[b]{2}{*}{ Ref. } \\
\hline & Material & Diameter $(\mathrm{nm})$ & Length $(\mu \mathrm{m})$ & & \\
\hline \multirow[t]{2}{*}{ HEK293 } & InAs & $70-270$ & $1-3$ & 1 & (Berthing, unpublished) \\
\hline & $\mathrm{Si}$ & 90 & $3-6$ & 1 & $(\mathrm{Kim}, 2007)$ \\
\hline $\mathrm{mES}$ & $\mathrm{Si}$ & $30-400$ & $3-6$ & $5-1$ & $(\mathrm{Kim}, 2007)$ \\
\hline Mouse nerve cells & $\mathrm{GaP}$ & 50 & 2.5 & 3 & (Hällström, 2007) \\
\hline $\mathrm{CHO}$ & $\mathrm{CNF}$ & $100-200$ & 10 & 22 & (McKnight, 2003, 2004) \\
\hline Mouse myeloma cells (non-adherent) & $\mathrm{CNF}$ & $100-200$ & 10 & 3 & (McKnight, 2004) \\
\hline Rat pheochromocytoma neurons & $\mathrm{CNF}$ & $100-200$ & 10 & 6 & (McKnight, Melechko, 2006) \\
\hline Rat hippocampal neurons & $\mathrm{CNF}$ & $100-200$ & 10 & 6 & (McKnight, Melechko, 2006) \\
\hline $\mathrm{HeLa}^{*}$ & CNT & 45 & - & 1 & (Chen, 2007) \\
\hline
\end{tabular}

*This study was not done on an array, but with a single CNT. It is included because it showed that cancer cells can be penetrated by CNTs. Length of CNT was not reported.

effects change with the dimensions and materials of the NWs (Jia et al., 2005) and are different for each cell type, so additional investigation need to be done in this field. It is also plausible that the toxic effects will be different when the wires are fixed to a surface, because there is not the same risk of formation of cytotoxic aggregates or accumulations of wires in intracellular compartments (Lacerda et al., 2007).

So far the viability of cells penetrated by fixed NWs has been evaluated based on rate of proliferation, membrane integrity, and respiratory activity. The membrane integrity (Hällström et al., 2007; Kim et al., 2007) and respiratory activity (Hällström et al., 2007) does not seem to be affected, but it is indicated that proliferation of mouse myeloma cells is slowed down upon interaction with carbon nanofibers (McKnight et al., 2004). Besides these simple viability tests, the effects of penetrating the intracellular environment are unknown. Before the potential of this platform can be fully exploited, it is necessary to determine the consequences of penetrating the cell membrane. For example, it will be important to investigate whether the NWs disturb the intracellular signaling pathways. Despite the outcome of future studies on NW toxicity it will be a substantial improvement if it is possible to remove the wires after they have fulfilled their purpose. This could be significantly simplified by using NWs fixed to a surface instead of NWs in solution.

Acknowledging that NW toxicity is still under investigation, but that initial studies show several cell types to be compatible with arrays of NWs of different materials, the possible applications of such a device will be discussed next.

\section{Delivery Platform for DNA, Particles, and Drugs}

Current therapies are limited not only by the identification of efficient potential therapeutic agents, but also by their inefficient translocation into the targeted cells. Indeed, because of the lipophilic nature of biological membranes, many biologically active compounds of different sizes need to be delivered inside the cell or even to the nucleus, to exert therapeutic action. Using arrays of NWs incorporated in cells with a controlled density and location, delivery of various types of molecules in the cell becomes possible (Fig. 3). A few examples of this application in vitro appeared recently (Chen et al., 2007; Kim et al., 2007) and clearly show the feasibility of using functionalized NWs for the release of compounds in cells.

The first applications of NW arrays concern DNA delivery. As shown by Kim et al., it was possible to coat arrays of silicon
NWs with DNA encoding GFP and use its fluorescence to estimate the efficiency of DNA transfection (2007). The protein was correctly expressed in the cells, indicating that the DNA was transferred from the wires to the nucleus. The limited transfection lefficiency tobserved can most probably be improved by delivering the DNA directly in the nucleus and increase the quantity of DNA delivered to the cell. This is likely to be achievable because it has already been shown that it is possible to penetrate the nuclear envelope with a nanoneedle maneuvered by an atomic force microscope (AFM) (Obataya et al., 2005). An advantage of using a NW array as a delivery platform compared to, for example, nanoparticles in solution is that the array provides spatial control of the delivery. This could be used, for example, for parallel delivery of several DNA constructs, while keeping track of which population of cells received what type of DNA.

The size of a DNA molecule being quite considerable, translocation of smaller sized molecules or particles, including proteins such as antibodies, can easily be predicted. Such application has not yet been demonstrated on arrays of NW, but the chemical functionalization of NWs with various chemical groups and proteins, including antibodies, is already possible (Liu, unpublished; Patolsky et al., 2006; Stern et al., 2007).

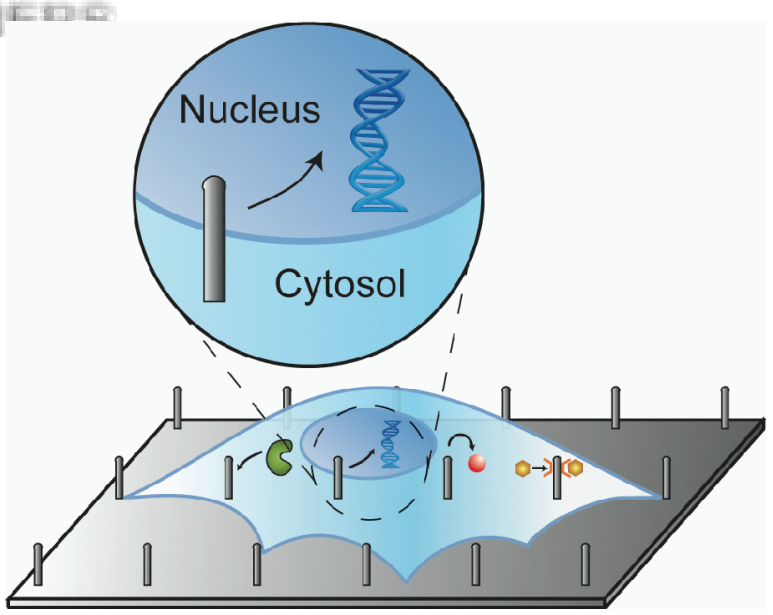

FIG. 3. Present and future applications of NW arrays interfaced with living cells: Delivery platform. Cell on a NW array illustrating the potential as delivery and detection platform for DNA (blue), particles (red, yellow) and proteins (green). NWs have small enough dimensions to enable direct delivery into the nucleus. 
When compounds are specifically immobilized on NWs, simple procedures to release the compounds in a cell can be used, as illustrated by Chen et al. They were able to deliver particles of $15 \mathrm{~nm}$ diameters into a cell using a CNT fixed to an AFM tip. The particles were connected to the CNT through disulfide bridges that were automatically cleaved in the reducing cytosol of the cell. A rewarding progress in this context would be the development of other specific cleavage modes for the release of compounds on demand.

The broad panel of compounds that can be attached to NWs (nanoparticles, DNA, and proteins such as antibodies) suggests a range of potential applications for diagnostic and drug discovery in cell cultures. In the future, this approach is also promising for the elaboration of novel therapies if detailed investigations reveal a poor toxicity of various types of NW arrays.

The delivery of compounds of different sizes and compositions at various locations in the cell using arrays of NWs has a high potential for the delivery of poorly permeable compounds, or for the delivery of compounds targeting specific cellular locations, such as nuclear receptors. This type of targeted delivery will considerably increase the efficiency of the compounds compared to current therapies using the same molecules (Gupta et al., 2005). As an example, peptides and antibodies are very high-potential cancer drugs that are easily degraded in the body before reaching the tumor cells. Using NW arrays after surgery to deliver these compounds into the remaining tumor cells will increase their efficiency but also contribute to a reduction of the side effects observed today due to their administration in large quantities and their toxic effect for healthy organs and tissues (Ferrari, 2005; Torchilin et al., 2003).

An additional interesting potential for arrays of NWs consists of the extraction of compounds from the cell. Such an approach would require not only a noninvasive incorporation of the NWs in the cell, but also a noninvasive extraction of the NWs. If successful, it would open significant prospective uses for diagnostics on biopsies.

\section{Arrays of NWs: A Biosensing Platform in Living Cells}

In the past decade, CNTs and NW-FETs have been developed into very sensitive label-free biosensors for applications in vitro (Cui et al., 2001; Patolsky et al., 2006). They were also recently shown to be used to sense cellular events (Patolsky et al., 2006; Stern et al., 2007) through modification of extracellular solutions. Therefore, NWs have already shown their potential within the field of biosensing. What NW arrays could add to this is the option of intracellular sensors.

Any cellular event is related to the modulation of the intracellular concentrations of cations and other messengers. There are increasing applications in cell imaging using such signals as an indicator of protein functionality (Lang et al., 2006). An example is the monitoring of modulation of calcium concentrations to report on the activity of $\mathrm{G}$ protein-coupled receptors, even if the increase of calcium cations in the cell is the product of a succession of reactions involving several proteins (Rudolf et al., 2003). The advantage of this downstream monitoring is already realized in drug discovery, with the development of assays in living cells rather than on isolated proteins, in order to integrate the biological complexity of cell signaling.
Current fluorescence-based sensors rely on the loading of cells with environment-sensitive fluorescent dyes. Some technical limitations regarding the efficiency and reproducibility of loading the dyes in the cell have been partly solved using translocated particles grafted with well-defined numbers of dyes (Wilson et al., 2007). Arrays of NWs can be used similarly to those particles because they can be functionalized with multiple compounds (Liu, unpublished; Patolsky et al., 2006; Stern et al., 2007) (Fig. 4). Moreover, as discussed earlier, the NWs can be made from different materials and doped with other elements or coated with protective or active layers; this opens up opportunities for the design of sensors with novel intrinsic properties to be directly exploited for cellular sensing.

The geometry of NWs and their immobilization on surfaces present two additional advantages compared to existing sensors: control of the number of sensors per cell and location of the sensors in the cell (Fig. 4), allowing a three-dimensional mapping of cellular events. This would especially be relevant within neuroscience for mapping signal propagation in a single nerve cell, but also in neuronal networks (Namiki et al., 2007; Wilson et al., 2005). The work of Hällström et al., indicates that even neuronal axons could be penetrated by NWs, which makes it interesting to investigate the potential of NW arrays for mapping signal propagation in fine networks of axons and dendrites.

\section{Electrophysiology in a Cell Using Arrays of Nanowires}

In 2006, McKnight et al., developed an array of individually contacted electrically active carbon nanofibers (CNF) on a silicon surface. This publication makes it reasonable to start looking into the application of NWs as nanoelectrodes in electrophysiology measurements (Fig. 5). With this device McKnight et al., succeeded in getting electrical responses from oxidative events occurring in intercellular regions of a neuronal culture that had been cultured directly on the nanofibers. The same type of array

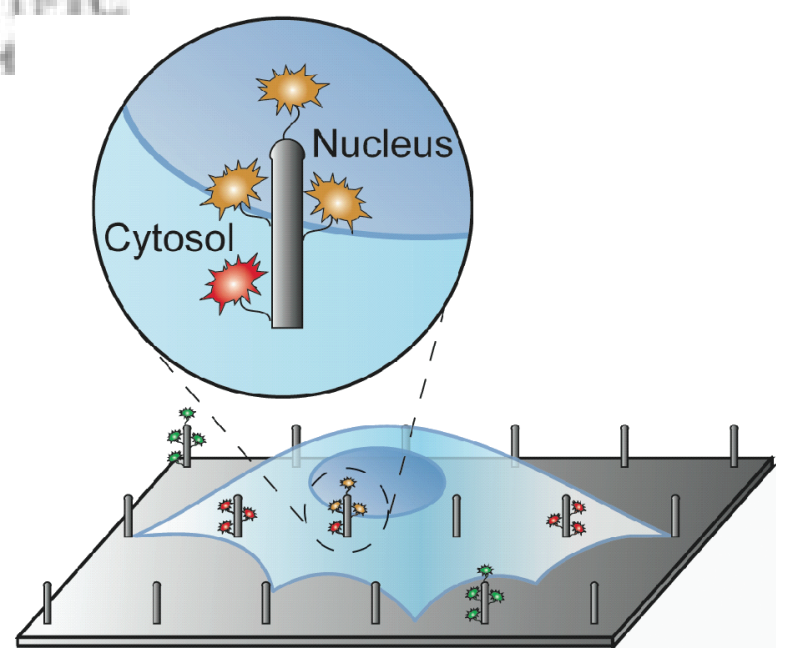

FIG. 4. Present and future applications of NW arrays interfaced with living cells: Biosensor. Cell on a NW array illustrating the potential as a fluorescence biosensor. NWs can be functionalized with environmentsensitive fluorophores (yellow, red, green) and provide spatially resolved information both within a single cell and in networks of cells. 


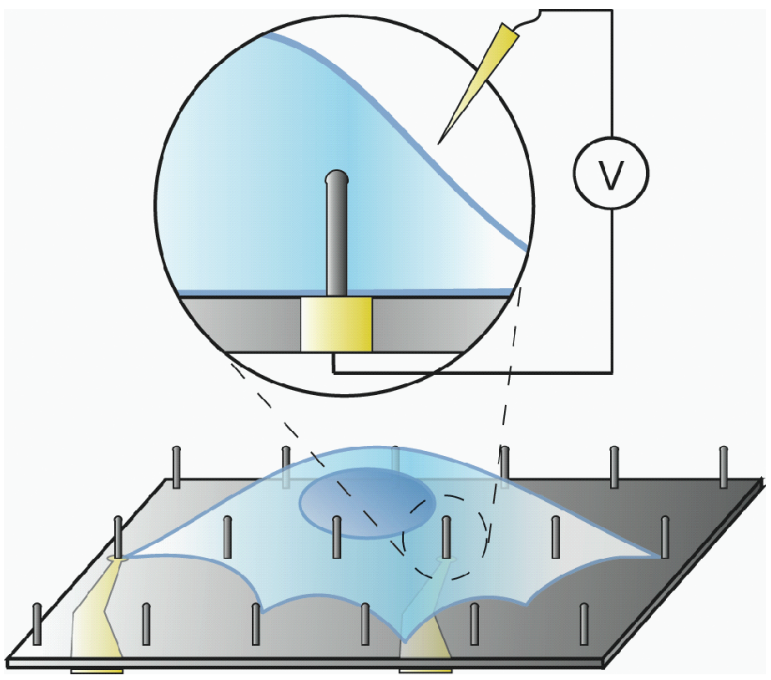

FIG. 5. Present and future applications of NW arrays interfaced with living cells: Electrophysiology. Cell on NW array illustrating the potential of NWs as intra- and extracellular nanoelectrodes in electrophysiology. Each NW can be electrically contacted through the surface (yellow). Changes in whole-cell potential are measured as a change in potential with respect to a standard-size extracellular counter electrode (yellow triangle). The array enables single-cell measurements on multiple cells simultaneously, representing future applications for HTS of drugs that target ion channels.

of CNFs has also been used for measuring electrophysiological activity in rat neural tissue slices (Yu et al., 2007).

A widely used tool in neuroscience is the patch clamp technique, used for measuring the activity of ion channels in the cell membrane. In traditional whole-cell patch clamp measurements intracellular access is attained by rupturing the cell membrane by means of applying suction pulses to a pipette attached to the cell membrane. After the cell membrane is broken, the saline solution in the pipette intermixes with the cytosol of the cell causing a change in the balance of the cell's intracellular solution. This change affects the function of ion channels and reduces the lifetime of the cell.

Standard laboratory patch clamp techniques still rely on the laborious use of a single glass patch pipette. This makes the testing of drug candidates acting on ion channels a very timeconsuming and expensive process. An automated method for performing high-quality patch clamp measurements is thus highly attractive for the pharmaceutical industry (Dunlop et al., 2008). In the last few years so-called planar chip-based patch clamp methods, suitable for high-throughput screening, have been developed (Asmild et al., 2003; QPatch 16 by Sophion Bioscience A/S). These systems typically consist of a surface having one or more holes (orifices) where cells are positioned by suction and electrophysiological measurements are performed. Although the planar patch clamp offers a less laborious operation than standard patch clamp, it still necessitates the same harmful rupture of the cell membrane.

The most appealing prospective of NW-electrodes would be their application as less invasive intracellular electrodes. If a high-resistance electrical seal can be obtained between the NW electrode and the extracellular buffer solution, highquality measurements of the cell membrane potential is possible. Such a "built-in" electrode would facilitate high-quality ultra high throughput (UHT) drug screening not possible with present state-of-the-art technology.

On the other hand, NW-electrodes might also pose a potential threat to the viability of the cells. Their electrochemical behavior inside the cell has so far not been investigated and one could imagine problems with, for example, gas generation at the electrodes when current is drawn from such NWs.

NWs could also be used advantageously in another area of electrophysiology; to replace "sharp glass electrodes." Sharp electrodes are used for monitoring the potential inside a cell. Due to their small tip size $(0.01-0.1 \mu \mathrm{m})$ the electrode resistance is often an order of magnitude higher $(>100 \mathrm{M} \Omega)$ than seen in normal patch pipettes. (The Axon CNS Guide, 2006). This high resistance causes a lot of electrical noise, which sets a limit to the sensitivity of the measurement. NW electrodes could offer a much lower electrode resistance and hence noise level, while at the same time substantially prolonging the cell lifetime.

In addition to the expected less invasive nature of the NWs, their small dimensions make it feasible to fabricate large arrays of NW electrodes, which can be addressed individually (Fig. 5). This would facilitate the incorporation of several electrodes in one cell, which would provide valuable information with a spatial resolution not possible by other means.

Furthermore, an array of NW electrodes could provide a visionary platform for probing electrophysiological properties not only of individual living cells, but also of networks of cells communicating; for example, neuronal networks. Understanding this signalling process and the flow of information between complex networks of cells would provide new, basic information crucial to areas like neuroscience, systems biology, and drug development.

\section{CONCLUSION}

NW arrays appear to be promising tools for nanomedicine, with a multitude of putative applications in drug delivery, diagnostics, and high-throughput screening of new molecules. The potential of arrays of NWs apply to a large range of cell types, with various adhesion properties, suggesting that such arrays can be adapted to a variety of cell cultures and tissues.

The first set of applications concerns in vitro investigation of cell cultures or isolated tissues for fundamental investigation of cellular signaling and cell/cell communication, but also the design of novel high-throughput screening strategies for new potentially therapeutic compounds or diagnostics on biopsies.

A second set of future applications concerns use of NW arrays in human tissue for innovative therapies. The combination of the properties of arrays of NWs with fine surgery presents a promising potential for local treatments, including tumor cells. Such applications will first require overcoming some technical limitations regarding the interface of cells with NWs, and a detailed investigation of their short- and long-term toxicity.

Acknowledgments: The authors thank Martin Aagesen, Nadia Cherouati, Lars Iversen, Erik Johnson, Loerke Münter Lassen, Jean-Baptiste Perez, Nathalie Rieben, Thomas Sand Jespersen, Brian Skov Sørensen and Dimitrios Stamou for discussions and critical reading of the manuscript. Financial support was provided by the Danish Research Council - FNU 272-050355, FNU 272-05-0358 \& NABIIT 2106-05-0036. 


\section{Abbreviations}

NW, nanowire; NWs, nanowires; CNT, carbon nanotube; HTS, high-throughput screening; FET, field-effect transistor; GFP, green fluorescent protein; $\mathrm{CHO}$, Chinese hamster ovary cells; HEK293, human embryonic kidney cells; mES, mouse embryonic stem cells; GaP, gallium-phosphide; InAs, indium-arsenide; AFM, atomic force microscope; CNF, carbon nanofiber; UHT, ultra high-throughput.

\section{REFERENCES}

Asmild M, Oswald N, Krzywkowski KM, Friis S, Jacobsen RB, Reuter D, Taboryski R, Kutchinsky J, Vestergaard RK, Schrøder RL, Sørensen CB, Bech M, Korsgaard MPG, Willumsen NJ (2003) Upscaling and automation of electrophysiology: Toward high-throughput screening in ion channel drug discovery. Receptors and Channels 9:49.

Berthing T, unpublished.

Cai D, Doughty CA, Potocky TB, Dufort FJ, Huang Z, Blair D, Kempa K, Ren ZF, Chiles TC (2007) Carbon nanotube-mediated delivery of nucleic acids does not result in non-specific activation of $\mathrm{B}$ lymphocytes. Nanotechnology 18:365101.

Chen X, Kis A, Zettl A, Bertozzi CR (2007) A cell nanoinjector based on carbon nanotubes. Proc Natl Acad Sci USA 104:8218-8222. Sc1el

Cheng MM-C, Cuda G, Bunimovich YL, Gaspari M, Heath JR, Hill HD, Mirkin CA, Nijdam AJ, Terracciano R, Thundat T, Ferrari M (2006) Nanotechnologies for biomolecular detection and medical diagnostics. Curr Opin Chem Biol 10:11-19.

Cui Y, Wei Q, Park H, Lieber CM (2001) Nanowire nanosensors for highly sensitive and selective detection of biological and chemical species. Science 293:1289-1292.

Dunlop J, Bowlby M, Peri R, Vasilyev D, Arias R (2008) Highthroughput electrophysiology: an emerging paradigm for ion-channel screening and physiology. Nat Rev Drug Discov 7:358-368.

Ferrari M (2005) Cancer nanotechnology: Opportunities and challenges. Nat Rev Cancer 5:161-171.

Guillorn MA, McKnight TE, Melechko AV, Merkulov VI, Britt PF, Austin DW, Lowndes DH, Simpson ML (2002) Individually addressable vertically aligned carbon nanofiber-based electrochemical probes. J Appl Phys 91:3824.

Gupta B, Levchenko TS, Torchilin VP (2005) Intracellular delivery of large molecules and small particles by cell-penetrating proteins and peptides. Adv Drug Deliv Rev 57:637-651.

Hällström W, Mårtensson T, Prinz C, Gustavsson P, Montelius L, Samuelson L, Kanje M (2007) Gallium phosphide nanowires as a substrate for cultured neurons. Nano Lett 7:2960-2965.

Jia G, Wang HF, Yan L, Wang X, Pei RJ, Yan T, Zhao YL, Guo XB (2005) Cytotoxicity of carbon nanomaterials: Single-wall nanotube, multi-wall nanotube, and fullerene. Environ Sci \& Technol 39:13781383.

Khang D, Lu J, Yao C, Haberstroh KM, Webster TJ (2007) The role of nanometer and sub-micron surface features on vascular and bone cell adhesion on titanium. Biomaterials 29:970-983.

Kim W, Ng JK, Kunitake ME, Conklin BR, Yang P (2007) Interfacing silicon nanowires with mammalian cells. J Am Chem Soc 129:72287229.

Kumar CSSR, ed (2007) Nanomaterials for Biosensors. Wiley-VCH.

Kunzler TP, Drobek T, Schuler M, Spencer ND (2007) Systematic study of osteoblast and fibroblast response to roughness by means of surfacemorphology gradients. Biomaterials 28:2175-2182.

Lacerda L, Raffa S, Prato M, Bianco A, Kostarelos K (2007) Cellpenetrating CNTs for delivery of therapeutics. Nano Today 2:38-43.

Lang P, Yeow K, Nichols A, Scheer A (2006) Cellular imaging in drug discovery. Nat Rev Drug Discovery 5:343-356.
Lauhon LJ, Gudiksen MS, Lieber CM (2004) Semiconductor nanowire heterostructures. Philosophical Transactions of the Royal Society of London Series A-Mathematical Physical and Engineering Sciences 362:1247-1260.

Li Y, Qian F, Xiang J, Lieber CM (2006) Nanowire electronic and optoelectronic devices. Mater Today 9:18-27.

Liu Y, unpublished.

Lu W, Lieber CM (2006) Semiconductor nanowires. J Physics D: Applied Physics R387.

Manna SK, Sarkar S, Barr J, Wise K, Barrera EV, Jejelowo O, RiceFicht AC, Ramesh GT (2005) Single-walled carbon nanotube induces oxidative stress and activates nuclear transcription factor-kappa B in human keratinocytes. Nano Lett 5:1676-1684.

Mårtensson T (2004) Nano Lett 5:761.

McKnight TE, Melechko AV, Hensley DK, Mann DGJ, Griffin GD, Simpson ML (2004) Tracking gene expression after DNA delivery using spatially indexed nanofiber arrays. Nano Lett 4:1213-1219.

McKnight TE, Melechko AV, Fletcher BL, Jones SW, Hensley DK, Peckys DB, Griffin GD, Simpson ML, Ericson MN (2006) Resident neuroelectrochemical interfacing using carbon nanofiber arrays. J Phys Chem B 110:15317-15327.

McKnight TE, Peeraphatdit C, Jones SW, Fowlkes JD, Fletcher BL, Klein KL, Melechko AV, Doktycz MJ, Simpson ML (2006) Sitespecific biochemical functionalization along the height of vertically aligned carbon nanofiber arrays. Chem Mater 18:3203-3211.

McKnight TE, Melechko AV, Griffin GD, Guillorn MA, Merkulov VI, Serna F, Hensley DK, Doktycz MJ, Lowndes DH, Simpson ML (2003) Intracellular integration of synthetic nanostructures with viable cells for controlled biochemical manipulation. Nanotechnology 14:551.

Microelectrodes and micropipettes, Chapter 4 (2006) In: The axon CNS guide to electrophysiology \& biophysics laboratory techniques. Molecular Devices.

Namiki S, Sakamoto H, Iinuma S, Iino M, Hirose K (2007) Optical glutamate sensor for spatiotemporal analysis of synaptic transmission. Eur J Neurosci 25:2249-2259.

Obataya I, Nakamura C, Han S, Nakamura N, Miyake J (2005) Nanoscale operation of a living cell using an atomic force microscope with a nanoneedle. Nano Lett 5:27-30.

Patolsky F, Zheng G, Lieber CM (2006) Nanowire sensors for medicine and the life sciences. Nanomed 1:51-65.

QPatch 16 by Sophion Bioscience A/S. www.sophion.dk.

Rudolf R, Mongillo M, Rizzuto R, Pozzan T (2003) Looking forward to seeing calcium. Nat Rev Mol Cell Biol 4:579-586.

Stern E, Klemic JF, Routenberg DA, Wyrembak PN, Turner-Evans DB,

Hamilton AD, LaVan DA, Fahmy TM, Reed MA (2007) Label-free immunodetection with CMOS-compatible semiconducting nanowires. Nature 445:519-522.

Thelander C, Agarwal P, Brongersma S, Eymery J, Feiner LF, Forchel A, Scheffler M, Riess W, Ohlsson BJ, Gösele U, Samuelson L (2006) Nanowire-based one-dimensional electronics. Mater Today 9:28-35.

Torchilin VP, Lukyanov AN (2003) Peptide and protein drug delivery to and into tumors: Challenges and solutions. Drug Discov Today 8:259-266.

Wilson GS, Ammam M (2007) In vivo biosensors. FEBS Journal 274:5452-5461.

Wilson GS, Gifford R (2005) Biosensors for real-time in vivo measurements. Biosen \& Bioelectron 20:2388-2403.

Wu Y, Fan R, Yang P (2002) Inorganic semiconductor nanowires. Int J Nano 1:1-39.

Yi C, Fong C-C, Chen W, Qi S, Lee S-T, Yang M (2007) Inhibition of biochemical reactions by silicon nanowires through modulating enzyme activities. Chembiochem 8:1225-1229.

Yu Z, McKnight T, Ericson M, Melechko A, Simpson M, Iii B (2007) Vertically aligned carbon nanofiber arrays record electrophysiological signals from hippocampal slices. Nano Lett 7:2188-2195. 